\title{
MODULES WITH PERFECT DECOMPOSITIONS
}

\author{
LIDIA ANGELERI HÜGEL and MANUEL SAORÍN*
}

It is well known that a module $M$ over an arbitrary ring admits an indecomposable decomposition whenever it has the property that every local direct summand of $M$ is a direct summand [28]. Recently, J. L. Gómez Pardo and P. Guil Asensio [18] have shown that requiring this property not only for $M$ but for any direct sum $M^{(\aleph)}$ of copies of $M$ even yields the existence of a decomposition of $M$ in modules with local endomorphism ring which, moreover, satisfies many nice properties of decompositions studied in the literature, like the exchange property, or the property of complementing direct summands. More precisely, it turns out that all these properties coincide if, instead of considering a single module $M$, we pass to the category Add $M$ of all direct summands of direct sums of copies of $M$.

In the present paper, we continue the investigation of these modules calling them modules with perfect decompositions. In Section 1, we show that a module $M$ has a perfect decomposition if and only if for every direct system $\left(M_{i}, f_{j i}\right)_{I}$ of modules in Add $M$ indexed by a totally ordered set $I$, the canonical epimorphism $\pi: \bigoplus_{i \in I} M_{i} \longrightarrow \lim M_{i}$ is a split epimorphism. This allows to shed a new light on a number of known examples of modules with perfect decomposition.

The remaining sections are devoted to the role played in this context by certain finiteness conditions over the endomorphism ring $S=$ End $M$. In fact, every module with a perfect decomposition is $S$-coperfect, that is, it satisfies the descending chain condition on cyclic $S$-submodules. Actually, in Section 2, we even show that $M$ is $\Sigma$-coperfect over $S$, i.e. any direct sum $M^{(\aleph)}$ of copies of $M$ is $S$-coperfect.

We thus discuss whether the converse implication also holds true. The best answer that we can give in full generality is the following (see Section 3): $\Sigma$ coperfectness over the endomorphism ring implies that the pure epimorphism

\footnotetext{
* The second author thanks the D.G.I. of the Spanish Ministry of Science and Technology and the Fundación "Séneca" of Murcia for their financial support.

Received July 29, 2004.
} 
$\pi: \bigoplus_{i \in I} M_{i} \longrightarrow \lim _{\longrightarrow} M_{i}$ associated to a direct system in Add $M$ as above, is even $\mathscr{C}$-pure where $\overrightarrow{\mathscr{C}}$ is the class of finitely generated $R$-modules.

We then focus on two main cases where being $\Sigma$-coperfect over $S$ is equivalent to the existence of a perfect decomposition. The first case is when $M$ is a direct sum of finitely generated modules. It is the topic of Section 4, where we also exhibit examples of modules with perfect decomposition related to the notion of pure-injectivity (4.4) or to tilting theory (4.6).

The second case, established in Section 5, is the case of CS (or extending) modules. Actually, here we can even prove that a CS-module has a perfect decomposition if and only if it is coperfect over its endomorphism ring. We close the paper with some examples relating our investigations to known results on decomposition of CS-modules.

\section{Perfect decompositions}

Let $R$ be an arbitrary ring, and let Mod $R$ be the category of all right $R$-modules. By a module $M$ we usually mean a right $R$-module, and we denote by Add $M$ the category consisting of all modules isomorphic to direct summands of direct sums of copies of $M$.

We start out by collecting some results on direct sum decompositions of $M$ which are scattered through the literature. First we have to recall some terminology.

A family $\left(N_{j}\right)_{j \in J}$ of submodules of a module $M_{R}$ is called independent when their sum is direct, i.e. when $N_{k} \cap \sum_{j \neq k} N_{j}=0$ for all $k \in J$. In such a case, $N=\oplus_{j \in J} N_{j}$ is called a local direct summand when $\oplus_{j \in F} N_{j}$ is a direct summand of $M_{R}$ for each finite subset $F \subseteq J$.

Moreover, a family of modules $\left(M_{i}\right)_{i \in I}$ is said to be locally semi-T-nilpotent if for each sequence of non-isomorphisms $M_{i_{1}} \stackrel{f_{1}}{\longrightarrow} M_{i_{2}} \stackrel{f_{2}}{\longrightarrow} M_{i_{3}} \ldots$, with pairwise different indices $\left(i_{n}\right)_{n \in \mathrm{N}}$ from $I$, and each element $x \in M_{i_{1}}$, there exists $m=m_{x} \in N$ such that $f_{m} f_{m-1} \ldots f_{1}(x)=0$. If the same condition is satisfied also when we allow repetitions in the sequence of indices $\left(i_{n}\right)_{n \in \mathrm{N}}$ involved, then the family $\left(M_{i}\right)_{i \in I}$ is called locally T-nilpotent.

Next, let $S$ be a ring with Jacobson radical $J(S)$. We say that a left module ${ }_{S} M$ is coperfect if it satisfies the descending chain condition for cyclic (or equivalently, finitely generated) $S$-submodules [8]. Furthermore, the ring $S$ is semiregular if $S / J(S)$ is von Neumann regular and idempotents lift modulo $J(S)$.

Finally, let us recall some properties of direct sum decompositions of modules. A module $M$ is said to have the exchange property if for any equality of the form $M^{\prime} \oplus A=\bigoplus_{l \in L} A_{l}$ with $M^{\prime} \cong M$ there exist submodules $B_{l} \subseteq A_{l}$ such that $M^{\prime} \oplus A=M^{\prime} \oplus \bigoplus_{l \in L} B_{l}$. Furthermore, a decomposition $M=\bigoplus_{k \in K} X_{k}$ 
is said to complement direct summands if for each direct summand $N$ of $M$ there is a subset $L \subseteq K$ such that $M=N \oplus \bigoplus_{k \in L} X_{k}$.

The following result subsumes classical and more recent results due to various authors.

THEOREM 1.1. The following statements are equivalent for a module $M$.

(1) Every local direct summand of a module in Add $M$ is a direct summand.

(2) $M=\bigoplus_{k \in K} X_{k}$, where $\left(X_{k}\right)_{k \in K}$ is a locally T-nilpotent family of indecomposable modules.

(3) $M$ has a decomposition in modules with local endomorphism ring, and $M$ is coperfect over its endomorphism ring.

(4) $M$ has a decomposition in modules with local endomorphism ring, and End $_{R} A$ is semiregular for all $A \in \operatorname{Add} M$.

(5) $M$ has an indecomposable decomposition, and every module in Add $M$ has the exchange property.

(6) Every module in Add $M$ has a decomposition that complements direct summands.

If these conditions are satisfied, we will say that $M$ has a perfect decomposition.

Proof. By $[18,2.3]$ it follows from condition (1) that $M$ has a decomposition in modules with local endomorphism ring. Moreover, it is shown in [22, Proposition E] that conditions (2) and (3) are equivalent. Then the equivalence of (1), (2) and (4) is a consequence of [21, 7.3.15], as shown in [2, 4.2]. For the equivalence of (1), (5) and (6), we refer to [18, 2.3].

We now want to characterize modules with perfect decompositions in terms of a property of direct limits. We collect here for later reference some wellknown facts about direct limits.

Lemma 1.2. Let I be a directed set and $\left(M_{i}, f_{j i}: M_{i} \rightarrow M_{j}\right)_{I}$ be a direct system in $\operatorname{Mod} R$. Denote by $\epsilon_{i}: M_{i} \rightarrow \bigoplus_{i \in I} M_{i}$ the canonical inclusion. For $i \leq j$ set $M_{j i}=M_{i}$ and consider the homomorphism $F: \bigoplus_{i \leq j} M_{j i} \longrightarrow$ $\bigoplus_{i \in I} M_{i}$ induced by the maps $\epsilon_{i}-\epsilon_{j} f_{j i}: M_{j i} \rightarrow \bigoplus_{i \in I} M_{i}$. Then the following hold true.

(1) There is an exact sequence $\bigoplus_{i \leq j} M_{j i} \stackrel{F}{\longrightarrow} \bigoplus_{i \in I} M_{i} \stackrel{\pi}{\longrightarrow} \underset{\lim }{\longrightarrow} M_{i} \rightarrow 0$ inducing a pure-exact sequence

$$
0 \longrightarrow \operatorname{Im}(F) \stackrel{\lambda}{\longrightarrow} \bigoplus_{i \in I} M_{i} \stackrel{\pi}{\longrightarrow} \underset{\lim }{\longrightarrow} M_{i} \longrightarrow 0
$$


(2) When I is infinite and totally ordered, $\operatorname{Im}(F)=\operatorname{Ker} \pi=\bigcup_{\alpha \in \mathscr{A}} N_{\alpha}$ where $\left(N_{\alpha}\right)_{\alpha \in \mathscr{A}}$ is a chain of direct summands of $\bigoplus_{i \in I} M_{i}$ which is indexed by a set $\mathscr{A}$ of the same cardinality as $I$.

Proof. (1) is well-known. For (2), we refer to[18, 2.1] where it is shown that the $N_{l}$ can be taken as $\sum_{i \leq j \leq l} \operatorname{Im}\left(\epsilon_{i}-\epsilon_{j} f_{j i}\right)$ with $l \in I$.

Proposition 1.3. Let $M$ be a module and $\aleph$ a cardinal. Then the following statements are equivalent.

(1) If $\left(N_{\alpha}\right)_{\alpha \in \mathscr{A}}$ is a chain of direct summands of $M^{(\aleph)}$ such that the cardinality of $\mathscr{A}$ is $\leq \aleph$, then the union $N=\bigcup_{\alpha \in \mathscr{A}} N_{\alpha}$ is a direct summand of $M^{(\aleph)}$.

(2) If $\left(M_{i}, f_{j i}\right)_{I}$ is a direct system where I is a totally ordered set of cardinality at most $\aleph$ and $M_{i}$ is isomorphic to a direct summand of $M^{(\aleph)}$ for all $i \in I$, then the canonical epimorphism $\pi: \bigoplus_{i \in I} M_{i} \longrightarrow \underline{\lim } M_{i}$ is a split epimorphism.

Proof. $(1) \Rightarrow(2)$ : follows from Lemma 1.2.

$(2) \Rightarrow(1)$ : We can assume w.l.o.g. that $\left(N_{i}\right)_{i \in I}$ is a chain of direct summands of $M^{(I)}$ where $I$ is a totally ordered set of cardinality $\aleph$. For each $i \in I$ we consider an idempotent $e_{i} \in$ End $M^{(I)}$ such that $\operatorname{Im}\left(e_{i}\right)=N_{i}$ and set $f_{i}=1-e_{i}$. In particular, we get $e_{i}(x)=x$ for all $x \in N_{i}$, and for $i \leq j$ we have $N_{i} \subseteq N_{j}$, hence $e_{j} e_{i}=e_{i}$ and $f_{j} f_{i}=f_{j}$. So, we can construct a direct system $\left(M_{i}, f_{j i}\right)_{I}$ by taking $M_{i}=M^{(I)}$ and $f_{j i}: M_{i} \rightarrow M_{j}$ with $f_{j i}=1_{M^{(I)}}$ if $i=j$ and $f_{j i}=f_{j}$ if $i<j$.

We adopt the notation of Lemma 1.2. By assumption, the exact sequence $0 \longrightarrow \operatorname{Im}(F) \stackrel{\lambda}{\longrightarrow} \bigoplus_{i \in I} M_{i} \stackrel{\pi}{\longrightarrow} \underset{\lim }{\longrightarrow} M_{i} \longrightarrow 0$ splits. So, there are homomorphisms $\rho: \bigoplus_{i \in I} M_{i} \longrightarrow \operatorname{Im}\left(\overrightarrow{F)}\right.$ and $u: \underline{\lim } M_{i} \longrightarrow \bigoplus_{i \in I} M_{i}$ such that $\rho \lambda=1_{\operatorname{Im}(F)}, \pi u=1_{\underline{\lim M_{i}}}$ and, moreover, $\lambda \rho \overrightarrow{+u} \pi=1_{\bigoplus_{i \in I} M_{i}}$.

Our aim is to show that the canonical surjection $v: M^{(I)} \rightarrow M^{(I)} / N$ is a split epimorphism.

We start out by constructing a homomorphism $\varphi: M^{(I)} / N \rightarrow \underline{\lim } M_{i}$. To this end, we fix an index $k \in I$, take the canonical map $\varphi_{k}=\pi \epsilon_{k}: M_{k} \longrightarrow$ $\stackrel{\lim }{\rightarrow} M_{i}$, and consider the composition $\varphi_{k} f_{k}: M^{(I)} \rightarrow \underline{\lim _{1}} M_{i}$. Note that by $\overrightarrow{\text { construction }} \varphi_{k} f_{k}=\varphi_{l} f_{l}$ for each $l \geq k$. But then, since for any element $x \in N$ there is an index $l_{0} \in I$ such that $x \in \operatorname{Ker} f_{l}$ for all $l \geq l_{0}$, it follows $\varphi_{k} f_{k}(N)=0$. This shows that $\varphi_{k} f_{k}$ induces a map $\varphi: M^{(I)} / N \rightarrow \underline{\lim } M_{i}$.

We now investigate the composition of the summation map $\nabla: \bigoplus_{i \in I} M_{i} \rightarrow$ $M^{(I)},\left(x_{i}\right)_{i \in I} \mapsto \sum_{i \in I} x_{i}$ with $u \varphi v$. Observe first that $\nabla F\left(M_{j i}\right) \subset \operatorname{Im} e_{j}$ for all $j \geq i$, hence $\operatorname{Im} \nabla \lambda \subset N$. So, if $y \in M^{(I)}$, we see that $\nabla u \varphi v(y)=$ $\nabla u \pi \epsilon_{k} f_{k}(y)=\nabla\left(1_{\oplus_{i \in I} M_{i}}-\lambda \rho\right)\left(\epsilon_{k} f_{k}(y)\right)=f_{k}(y)-n$ for some $n \in N$. 
As $y-f_{k}(y)=e_{k}(y) \in N$, we infer $v \nabla u \varphi v(y)=v(y)$. Since $v$ is an epimorphism, this shows that $v \nabla u \varphi$ is the identity map and, hence, $v$ is a split epimorphism.

As a consequence, we obtain a new characterization of modules with perfect decompositions. In the proof, the term totally ordered direct limit means that the underlying directed index set is totally ordered.

THEOREM 1.4. The following statements are equivalent for a module $M$.

(1) M has a perfect decomposition.

(2) If $\left(M_{i}, f_{j i}\right)_{I}$ is a direct system such that I is a totally ordered set and $M_{i} \in \operatorname{Add} M$ for all $i \in I$, then the canonical epimorphism $\pi: \bigoplus_{i \in I} M_{i} \longrightarrow \lim _{\longrightarrow} M_{i}$ is a split epimorphism.

(3) Every direct limit of split monomorphisms in Add $M$ is a split monomorphism in Add $M$.

Proof. By Theorem 1.1 and [28, 2.16], condition (1) means that the union of every chain of direct summands of a module in Add $M$ is a direct summand. The equivalence $(1) \Leftrightarrow(2)$ is thus an immediate consequence of Proposition 1.3 and Lemma 1.2.

In order to prove $(3) \Rightarrow(1)$, take a chain $\left(N_{i}\right)_{i \in I}$ of direct summands of a module $X \in \operatorname{Add} M$. Then the sequence $0 \rightarrow \bigcup_{i \in I} N_{i} \hookrightarrow X \rightarrow X / \bigcup_{i \in I} N_{i}$ $\rightarrow 0$ is a direct limit of split exact sequences in Add $M$, whence it is split-exact and, by [28, 2.16] again, assertion (1) follows.

We finally prove $(2) \Rightarrow(3)$. Since the case when $I$ is finite is trivial, we assume, without loss of generality, that $I$ is infinite. We need to prove that if $f:\left(X_{i}\right)_{i \in I} \longrightarrow\left(Y_{i}\right)_{i \in I}$ is a morphism of direct systems in Add $M$ such that $f_{i}: X_{i} \longrightarrow Y_{i}$ is a split monomorphism for every $i \in I$, then the induced morphism $\underset{\lim }{\longrightarrow} X_{i} \longrightarrow \underset{\lim }{\longrightarrow} Y_{i}$ is a split monomorphism in Add $M$.

It is not restrictive to assume that $I$ is totally ordered. Indeed, it is known that if $I$ has cardinality $\operatorname{card}(I)=\lambda$, then there is a chain $\left(I_{\kappa}\right)_{\kappa<\lambda}$ of directed subsets of $I$ such that $I=\bigcup_{\kappa<\lambda} I_{\kappa}$ and $\operatorname{card}\left(I_{\kappa}\right)<\lambda$ for every $\kappa$. By transfinite induction on $\lambda$, we suppose the result is true when the underlying directed set has cardinality $<\lambda$. Then the induced morphism $X(\kappa)=: \lim _{i \in I_{\kappa}} X_{i} \longrightarrow$ $\lim _{i \in I_{\kappa}} Y_{i}=: Y(\kappa)$ is a split monomorphism in Add $M$ for every $\kappa<\lambda$. But $\lim _{\longrightarrow}(f): \lim _{i \in I} X_{i} \longrightarrow \lim _{i \in I} Y_{i}$ coincides with the induced morphism $\lim _{\kappa<\lambda} X(\kappa) \longrightarrow \lim _{(\rightarrow<\lambda} Y(\kappa)$, which is a totally ordered direct limit of split monomorphisms in Add $M$ by the induction hypothesis. This proves that the problem is reduced to the case in which $I$ is totally ordered.

So, for the rest of the proof, we assume that $I$ is totally ordered. Let $0 \rightarrow$ $\left(X_{i}\right) \longrightarrow\left(Y_{i}\right) \longrightarrow\left(Z_{i}\right) \rightarrow 0$ be the corresponding short exact sequence of 
direct systems in Add $M$, so that $0 \rightarrow X_{i} \longrightarrow Y_{i} \longrightarrow Z_{i} \rightarrow 0$ is split-exact for all $i \in I$. We consider the commutative diagram with exact rows:

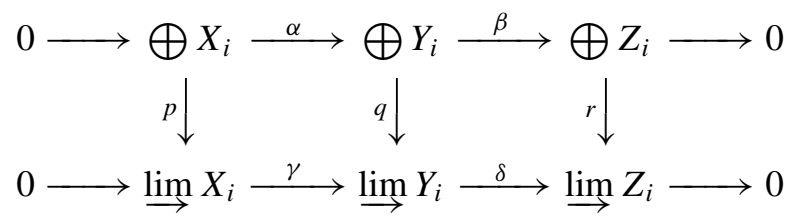

where the morphisms are the obvious ones. Since by hypothesis $r$ and $\beta$ are split epimorphism, the same is true for $r \beta=\delta q$, from which we get that $\delta$ is a split epimorphism as desired.

EXAMPLES 1.5. (1) Every $\Sigma$-pure-injective module has a perfect decomposition [22, Proposition E]. More generally, $M$ has a perfect decomposition if it is $\Sigma$-pure-split, i.e. every pure submodule of a direct sum of copies of $M$ is a direct summand. Indeed, in this case condition (2) of Theorem 1.4 is satisfied since $\operatorname{Ker} \pi$ is a pure submodule of $\bigoplus_{i \in I} M_{i}$.

(2) If $M$ is finitely generated, then it has a perfect decomposition if and only if $S=$ End $M$ is a right perfect ring [1, 29.5].

(3) If $M$ is a direct sum of finitely presented modules, then it has a perfect decomposition if and only if the class Add $M$ is closed under direct limits [2, 4.4]. This can be seen here as a direct consequence of Theorem 1.4.

(4) Recall that $\operatorname{Pext}_{R}^{1}(-,-)$ is the sub-bifunctor of $\operatorname{Ext}_{R}^{1}(-,-)$ formed by taking the pure-exact sequences. Let $\mathscr{A}$ be a class of $R$-modules closed under direct limits (and thus also closed under direct summands, cf. [13, proof of Lemma 1]), and $\mathscr{B}=\left\{B \in \operatorname{Mod} R \mid \operatorname{Pext}_{R}^{1}(A, B)=0\right.$ for all $\left.A \in \mathscr{A}\right\}$. If $M^{(\aleph)}$ belongs to $\mathscr{A} \cap \mathscr{B}$ for every cardinal $\aleph$, then $M$ has a perfect decomposition.

Indeed, if $X=\bigoplus_{\alpha \in \Lambda} X_{\alpha}$ is a local direct summand of $M^{(\aleph)}$, then the canonical sequence $0 \rightarrow X \hookrightarrow M^{(\aleph)} \longrightarrow M^{(\aleph)} / X \rightarrow 0$ is the direct limit of the split-exact sequences $0 \rightarrow X_{F} \hookrightarrow M^{(\aleph)} \longrightarrow M^{(\aleph)} / X_{F} \rightarrow 0$, where $X_{F}=\bigoplus_{\alpha \in F} X_{\alpha}$ for every finite subset $F \subset \Lambda$. We have $X \in$ Add $M \subseteq$ $\mathscr{B}$ and, since $\mathscr{A}$ is closed for direct limits, we also get that $M^{(\aleph)} / X \in \mathscr{A}$. Consequently, the pure-exact sequence $0 \rightarrow X \hookrightarrow M^{(\aleph)} \longrightarrow M^{(\aleph)} / X \rightarrow 0$ is split-exact. Then every local direct summand of $M^{(\aleph)}$ is a direct summand, and therefore $M$ has a perfect decomposition.

Particular cases of this situation are when $M_{R}$ is a tilting module in the sense of [3] such that Add $M$ is closed for direct limits (take $\mathscr{A}=\operatorname{Add} M$ ), and the case when $M_{R}$ is flat and $\Sigma$-cotorsion (take for $\mathscr{A}$ the class of flat $R$-modules). Hence, we rediscover, in a more general form, a recent result of Guil-Asensio and Herzog [20, Prop. 7, Theorem 8]. 
(5) Let $M_{R}$ be a classical tilting module in the sense of [10], i.e. $M_{R}$ is a finitely presented tilting module of projective dimension at most one. Let further $S=$ End $M$, and denote by $(\mathscr{A}, \mathscr{B})$ the cotorsion pair cogenerated by $M$, that is, $\mathscr{B}=M^{\perp}=\left\{X_{R} \mid \operatorname{Ext}_{R}^{1}(M, X)=0\right\}$ and $\mathscr{A}=\perp \mathscr{B}=\left\{X_{R} \mid\right.$ $\operatorname{Ext}_{R}^{1}(X, B)=0$ for all $\left.B \in \mathscr{B}\right\}$. Then $M_{R}$ has a perfect decomposition if and only if the class $\mathscr{A}$ is closed under direct limits.

In fact, note that $\mathscr{B}$ is always closed under direct limits. So, if $\mathscr{A}$ is closed under direct limits, the same holds true for Add $M=\mathscr{A} \cap \mathscr{B}$, and $M$ has a perfect decomposition by Example (4). Conversely, assume that $M$ has a perfect decomposition. Since $M$ is finitely presented, we then know from [2, 4.4] that every pure submodule of a module in Add $M$ is a direct summand. We proceed as in the proof of $[4,4.2]$. We first show that every module $X \in$ $\mathscr{B}$ which is a direct limit of modules from $\mathscr{A}$ admits a pure-exact sequence $0 \longrightarrow B \longrightarrow A \longrightarrow X \longrightarrow 0$ with $B \in \mathscr{B}$ and $A \in$ Add $M$ and therefore belongs to Add $M$. From this we deduce that $\mathscr{A}$ is closed under direct limits.

(6) Every $\Sigma$-CS-module has a perfect decomposition. This is shown by J. L. Gómez Pardo and P. Guil Asensio in [19, 2.4] and [18, 2.3]. We will see in Section 5 that a CS-module has a perfect decomposition if and only if it is coperfect over its endomorphism ring.

Further examples will be discussed in Section 4.

\section{Modules which are $\Sigma$-coperfect over their endomorphism ring}

We know from Theorem 1.1 that every module with a perfect decomposition is coperfect over its endomorphism ring. We now want to investigate more thoroughly the role played by endocoperfectness in this context. To this end we need to consider a stronger condition. Given a ring $S$ and a positive integer $r$, we will say that a left $S$-module $M$ is $r$-coperfect if every direct sum of at most $r$ copies of $M$ is coperfect. Moreover, we will say that $M$ is $\Sigma$-coperfect if $M$ is $r$-coperfect for all $r \in \mathrm{N}$.

In order to relate these notions to perfect decompositions, we will need the following result on countable direct limits. Related results can be found in [33] and in [24], [30], [31], [5].

Proposition 2.1. Let $\left(M_{n}\right)_{n \in \mathrm{N}}$ be a countable family of modules and let $M_{1} \stackrel{f_{1}}{\longrightarrow} M_{2} \stackrel{f_{2}}{\longrightarrow} M_{3} \stackrel{f_{3}}{\longrightarrow} \ldots$. be a sequence of homomorphisms. Consider the direct system $\left(M_{n}, f_{n m}\right)_{\mathrm{N}}$ given by $f_{n m}=1_{M_{n}}$ if $n=m$ and $f_{n m}=f_{n-1} \ldots f_{m}$ if $n>m$. Then the canonical epimorphism $\pi: \bigoplus_{n \in \mathrm{N}} M_{n} \longrightarrow \lim _{\longrightarrow} M_{n}$ splits if and only if there is a sequence of homomorphisms ... $\stackrel{g_{3}}{\longrightarrow} M_{3} \stackrel{g_{2}}{\longrightarrow} M_{2} \stackrel{g_{1}}{\longrightarrow}$ $M_{1}$ with the following property: 
For each index $m \in \mathbf{N}$ and each finite subset of elements $X=\left\{x_{1}, \ldots, x_{r}\right\}$ $\subseteq M_{m}$ there is an index $l=l(m, X)>m$ such that $f_{n m}(x)=g_{n} f_{n+1, m}(x)$ for all $n \geq l$ and all $x \in X$.

Proof. Let $F$ be as in Lemma 1.2. Observe that $\operatorname{Im} F=\operatorname{Im}(1-f)$ where $1=1_{\bigoplus_{n \in \mathrm{N}} M_{n}}$ and $f \in$ End $\bigoplus_{n \in \mathrm{N}} M_{n}$ is given by the matrix

$$
\left(\begin{array}{cccc}
0 & \ldots & & \\
f_{1} & 0 & \ldots & \\
0 & f_{2} & 0 & \ldots \\
\vdots & 0 & f_{3} & \ddots \\
& \vdots & \ddots & \ddots
\end{array}\right)
$$

So, $\pi$ splits if and only if $\operatorname{Im}(1-f)$ is a direct summand of $\bigoplus_{n \in \mathrm{N}} M_{n}$, and the only-if-part of the statement is shown in [39, Lemma 5]. For the if-part, we define an endomorphism $g \in$ End $\bigoplus_{n \in \mathbb{N}} M_{n}$ by the matrix $\left(g_{i j}\right)_{i, j \in \mathrm{N}}$ with

$$
g_{i j}: M_{j} \rightarrow M_{i}, g_{i j}= \begin{cases}-g_{i} & \text { if } j=i+1 \\ \left(-g_{i}\right)\left(-g_{i+1}\right) \ldots\left(-g_{j-1}\right) & \text { if } j>i+1 ; \\ 1_{M_{i}}-g_{i} f_{i} & \text { if } j=i \\ \left(1_{M_{i}}-g_{i} f_{i}\right) f_{i-1} \ldots f_{j} & \text { if } j<i .\end{cases}
$$

Let us verify that $g$ is well-defined: If $m \in \mathrm{N}$ and $x \in M_{m}$, then we can interpret $g(x)$ as the vector whose entries are the homomorphisms in the $m$-th column of $\left(g_{i j}\right)$ applied on the element $x$. So, the entries with index $n>m$ have the form $\mathrm{pr}_{M_{n}} g(x)=g_{n m}(x)=\left(1_{M_{n}}-g_{n} f_{n}\right) f_{n-1} \ldots f_{m}(x)=f_{n m}(x)-g_{n} f_{n+1, m}(x)$. Thus we know by assumption that there is an index $l=l(m, x) \in \mathrm{N}$ such that $\operatorname{pr}_{M_{n}} g(x)=0$ for all $n \geq l$, and we conclude that $g(x) \in \bigoplus_{n \in \mathrm{N}} M_{n}$.

We now claim that $g(1-f)$ is an isomorphism. In fact, the $(i, j)$-th entry of the matrix representing $g(1-f)$ is $g_{i j}-g_{i, j+1} f_{j}$, which equals $\left(1_{M_{i}}-g_{i} f_{i}\right)+$ $g_{i} f_{i}=1_{M_{i}}$ if $i=j$, and equals zero if $i>j$. This shows that $g(1-f)=1-h$ where $h \in$ End $\bigoplus_{n \in \mathrm{N}} M_{n}$ is represented by an upper triangular matrix. Since $h$ is then a locally nilpotent endomorphism of $\bigoplus_{n \in \mathrm{N}} M_{n}$, the sum $\sum_{n \in \mathrm{N}} h^{n}$ defines an endomorphism of $\bigoplus_{n \in \mathrm{N}} M_{n}$ which is inverse to $g(1-f)$.

This proves that $1-f$ is a split monomorphism and completes the proof.

Let us now show that modules which are $\Sigma$-coperfect over their endomorphism ring are characterized by a "local version" of the property considered above.

Proposition 2.2. Let $M$ be a module with $S=$ End $M$, and let $r$ be a positive integer. Then the following statements are equivalent. 
(1) ${ }_{S} M$ is $r$-coperfect.

(2) If $X \subseteq M$ is a subset consisting of at most $r$ elements, then the left $S$-module $S / \operatorname{ann}_{S}(X)$ is coperfect.

(3) If $M \stackrel{f_{1}}{\longrightarrow} M \stackrel{f_{2}}{\longrightarrow} M \stackrel{f_{3}}{\longrightarrow} \ldots$ is a sequence of endomorphisms and $f_{n m}=f_{n-1} \ldots f_{m}$ for $n>m$, then the following condition is satisfied:

For each index $m \in \mathrm{N}$ and each subset $X \subseteq M$ consisting of at most $r$ elements there are a sequence of endomorphisms . . $\stackrel{g_{3}}{\longrightarrow} M \stackrel{g_{2}}{\longrightarrow} M \stackrel{g_{1}}{\longrightarrow}$ $M$ and an index $l=l(m, X) \in \mathbf{N}$ such that $f_{n m}(x)=g_{n} f_{n+1, m}(x)$ for all $n \geq l$ and all $x \in X$.

Proof. (1) $\Rightarrow(2)$ : Use the $S$-linear embedding $S / \operatorname{ann}_{S}\left(\left\{x_{1}, \ldots, x_{r}\right\}\right) \longrightarrow$ $M^{r}, \bar{f} \mapsto\left(f\left(x_{1}\right), \ldots, f\left(x_{r}\right)\right)$.

(2) $\Rightarrow$ (3): Fix $m \in N$ and $X=\left\{x_{1}, \ldots, x_{r}\right\} \subseteq M$, and consider the descending chain of cyclic $S$-submodules of ${ }_{S} \bar{S}=S / \operatorname{ann}_{S}(X)$

$$
\overline{S f_{m}} \supseteq \overline{S f_{m+1} f_{m}} \supseteq \ldots
$$

By assumption there is an index $l \in \mathrm{N}$ such that $\overline{S f_{n-1} \ldots f_{m}}=\overline{S f_{n} \ldots f_{m}}$ for all $n \geq l$. Hence for each $n \geq l$ there is $g_{n} \in S$ such that $\overline{f_{n m}}=\overline{g_{n} f_{n+1, m}}$, that is, $f_{n m}\left(x_{i}\right)=g_{n} f_{n+1, m}\left(x_{i}\right)$ for all $1 \leq i \leq r$. Then the sequence $g_{1}=$ $0, g_{2}=0, \ldots, g_{l-1}=0, g_{l}, g_{l+1}, \ldots$ has the stated properties.

(3) $\Rightarrow(1)$ : Let us consider a descending chain of cyclic $S$-submodules of $M^{r}$

$$
S \underline{x} \supseteq S f_{1} \underline{x} \supseteq S f_{2} f_{1} \underline{x} \supseteq \ldots
$$

with $\underline{x}=\left(x_{1}, \ldots, x_{r}\right) \in M^{r}$ and $f_{1}, f_{2}, \ldots \in S$. By assumption there are an index $l=l(\underline{x}) \in \mathrm{N}$ and a sequence of endomorphisms $g_{1}, g_{2}, \ldots$ of $M$ such that $f_{n 1}\left(x_{i}\right)=g_{n} f_{n+1,1}\left(x_{i}\right)$ for all $n \geq l$ and all $1 \leq i \leq r$. Since $S$ acts componentwise on the elements of $M^{r}$, this means that $f_{n-1} \ldots f_{1} \underline{x} \in$ $S f_{n} \ldots f_{1} \underline{x}$ for all $n \geq l$, so our chain is stationary.

Combining Theorem 1.4 with Propositions 2.1 and 2.2 we obtain

Corollary 2.3. If a module $M$ has a perfect decomposition, then $M$ is $\Sigma$-coperfect over its endomorphism ring.

The above investigations rise the following questions.

Question 1. Let $M$ be a module which is $\Sigma$-coperfect over its endomorphism ring. Does it have a perfect decomposition?

Question 2. Let $M_{R}$ be a module which is coperfect over $S=$ End $M$. Is it $\Sigma$-coperfect over $S$ ? 
We have not been able to answer these questions in full generality, although some partial answers will be given in the sequel.

We start with a discussion of Question 2. First of all, note that in general, direct sums of coperfect modules need not be coperfect, see [9, Example 3]. However, as a consequence of Theorem 1.1 and Corollary 2.3, we obtain

COROLlary 2.4. Let $M$ be a module having a decomposition in modules with local endomorphism ring, and let $S=$ End $M$. If ${ }_{S} M$ is coperfect, then ${ }_{S} M$ is even $\Sigma$-coperfect.

Moreover, we next see that endocoperfectness is preserved under taking (direct sum) powers.

Proposition 2.5. Let $S$ be a ring, and $M$ a left $S$-module. Then $M$ is coperfect over $S$ if and only if for every index set $I$ the direct sum $M^{(I)}$ is coperfect over the ring $\mathrm{CFM}_{I}(S)$ of column-finite $I \times I$-matrices over $S$.

Proof. Let $I$ be a set, $A=\operatorname{CFM}_{I}(S)$. Consider a descending chain of cyclic $A$-submodules of $M^{(I)}$

$$
A x \supseteq A a_{1} x \supseteq A a_{2} a_{1} x \supseteq \ldots
$$

with $x \in M^{(I)}$ and $a_{1}, a_{2}, \ldots \in A$. Note that $x$ is contained in a finite subsum $M^{r_{1}}$ of $M^{(I)}$. Similarly, $a_{1} x$ is contained in a finite subsum $M^{r_{2}}$ of $M^{(I)}$, and so on. We thus only need to consider suitable $r_{n+1} \times r_{n}$-submatrices $\widetilde{a_{n}}$ of $a_{n}$, and have to find an index $l=l(X) \in \mathrm{N}$ and matrices $\widetilde{b_{n}} \in S^{r_{n} \times r_{n+1}} \subset A, n \geq l$, such that $\widetilde{a}_{n-1} \ldots \tilde{a_{1}} x=\tilde{b_{n}} \widetilde{a_{n}} \ldots \tilde{a}_{1} x$ for all $n \geq l$.

For each $n \in \mathrm{N}$ we write $\widetilde{a_{n 1}}(x)={\widetilde{a_{n-1}}}_{n} \ldots{\tilde{a_{1}}} x$ as vector and $\widetilde{a_{n}}$ as matrix as follows:

$$
\begin{gathered}
\widetilde{a_{n 1}}(x)=\left(\begin{array}{c}
y_{1}(n) \\
\vdots \\
y_{r_{n}}(n)
\end{array}\right) \in M^{r_{n}}, \\
\widetilde{a_{n}}=\left(\begin{array}{ccc}
a_{11}(n) & \ldots & a_{1, r_{n}}(n) \\
\vdots & & \vdots \\
a_{r_{n+1}, 1}(n) & \ldots & a_{r_{n+1}, r_{n}}(n)
\end{array}\right) \in S^{r_{n+1} \times r_{n}}
\end{gathered}
$$

Then we have the relations

$$
\left(\begin{array}{c}
y_{1}(n+1) \\
\vdots \\
y_{r_{n+1}}(n+1)
\end{array}\right)=\left(\begin{array}{ccc}
a_{11}(n) & \ldots & a_{1, r_{n}}(n) \\
\vdots & & \vdots \\
a_{r_{n+1}, 1}(n) & \ldots & a_{r_{n+1}, r_{n}}(n)
\end{array}\right)\left(\begin{array}{c}
y_{1}(n) \\
\vdots \\
y_{r_{n}}(n)
\end{array}\right)
$$


showing that $y_{1}(n+1), \ldots, y_{r_{n+1}}(n+1) \in \sum_{1 \leq k \leq r_{n}} S y_{k}(n)$. In other words, we have a descending chain of finitely generated submodules of ${ }_{S} M$

$$
\sum_{1 \leq k \leq r_{1}} S y_{k}(1) \supseteq \sum_{1 \leq k \leq r_{2}} S y_{k}(2) \supseteq \ldots
$$

which is stationary by a well-known result of Björk [8]. Thus there is an index $l \in \mathrm{N}$ such that $\sum_{1 \leq k \leq r_{n}} S y_{k}(n)=\sum_{1 \leq k \leq r_{n+1}} S y_{k}(n+1)$ for all $n \geq l$. But then for each $n \geq l$ we can write

$$
\left(\begin{array}{c}
y_{1}(n) \\
\vdots \\
y_{r_{n}}(n)
\end{array}\right)=\left(\begin{array}{ccc}
b_{11}(n) & \ldots & b_{1, r_{n+1}}(n) \\
\vdots & & \vdots \\
b_{r_{n}, 1}(n) & \ldots & b_{r_{n}, r_{n+1}}(n)
\end{array}\right)\left(\begin{array}{c}
y_{1}(n+1) \\
\vdots \\
y_{r_{n+1}}(n+1)
\end{array}\right)
$$

for suitable $b_{k j}(n) \in S$. This gives rise to the desired matrices $\widetilde{b_{n}} \in S^{r_{n} \times r_{n+1}}$.

COROllary 2.6. Let $R$ be a ring, $M$ be a right $R$-module with $S=$ $\operatorname{End}_{R}(M)$, and $r>0$ an integer. The following assertions are equivalent:

(1) ${ }_{S} M$ is $r$-coperfect.

(2) $M^{(I)}$ is $r$-coperfect over $\operatorname{End}_{R}\left(M^{(I)}\right)$ for every index set $I$.

(3) $M^{(I)}$ is $r$-coperfect over $\mathrm{CFM}_{I}(S)$ for every index set $I$.

Proof. (1) $\Leftrightarrow$ (3) follows from Proposition 2.5 bearing in mind that $\left(M^{(I)}\right)^{r}$ $\cong\left(M^{r}\right)^{(I)}$ as left $\mathrm{CFM}_{I}(S)$-modules, and $(2) \Rightarrow(1)$ is clear.

$(3) \Rightarrow(2)$ : Clearly, $A=: \operatorname{CFM}_{I}(S)$ is a subring of $T=: \operatorname{End}_{R}\left(M^{(I)}\right)$. Moreover, for every $x \in\left(M^{(I)}\right)^{r}$ and every $f \in T$, there is a $g \in A$ such that $g(x)=f(x)$. If now $T x \supseteq T f_{1}(x) \supseteq T f_{2} f_{1}(x) \ldots$ is a descending chain of cyclic $T$-submodules of $\left(M^{(I)}\right)^{r}$, then we can succesively replace $f_{i}$ by $g_{i} \in A$ so that $g_{i} \ldots g_{1}(x)=f_{i} \ldots f_{1}(x)$ for $i=1,2, \ldots$ Hence we get a descending chain $A x \supseteq A g_{1}(x) \supseteq A g_{2} g_{1}(x) \supseteq \ldots$ of cyclic $A$-submodules which is stationary under the hypothesis (3), so that there exist $k>0$ and a sequence of elements $h_{n} \in A, n \geq k$, such that $h_{n} g_{n}\left(g_{n-1} \ldots g_{1}(x)\right)=g_{n-1} \ldots g_{1}(x)$ for all $n \geq k$. But then $h_{n} f_{n}\left(f_{n-1} \ldots f_{1}(x)\right)=f_{n-1} \ldots f_{1}(x)$ and since $h_{n} \in$ $A \subseteq T$, we conclude that $T f_{n} f_{n-1} \ldots f_{1}(x)=T f_{n-1} \ldots f_{1}(x)$ for all $n \geq k$.

We end this section by considering the following aspect of Question 2:

REMARK 2.7. Let $R$ be a ring. The following assertions hold true.

(1) $\Sigma$-coperfectness over the endomorphism ring is a Morita invariant property.

(2) Coperfectness over the endomorphism ring is a Morita invariant property for $R$-modules if and only if every endocoperfect $R$-module is $\Sigma$ coperfect over its endomorphism ring. 
Proof. (1) A module is $\Sigma$-coperfect over $S=\operatorname{End}_{R} M$ if and only if condition (3) of Proposition 2.2. holds true for every finite subset $X \subseteq M$, or equivalently, for every finitely generated submodule $X$ of $M_{R}$. The latter property is clearly Morita invariant.

(2) The if-part follows immediately from (1). For the only-if-part, take an endocoperfect module $M_{R}$, and let $S=\operatorname{End}_{R} M$. The canonical Morita equivalence Mod $R \stackrel{\cong}{\cong}$ Mod $R^{r \times r}$ takes $M_{R}$ to the endocoperfect $R^{r \times r}$-module $M^{r}$, whose endomorphism ring is also $S$. Then ${ }_{S} M^{r}$ is coperfect for every $r>0$, so that ${ }_{S} M$ is $\Sigma$-coperfect.

\section{Endocoperfectness and purity}

We now come back to Question 1. Given a module which is $\Sigma$-coperfect over its endomorphism ring, how far is it from having a perfect decomposition?

We first compare endocoperfectness with $\Sigma$-pure-injectivity. To this end, we use that a module $M$ is $\Sigma$-pure-injective if and only if it satisfies the descending chain condition on (finite) matrix subgroups [38]. Recall that, if $Y_{R}$ is a module and $U$ a subgroup of the abelian group $Y$, then $U$ is said to be a matrix subgroup of $Y$ if there is a module $A_{R}$ and an element $x \in A$ such that $U$ equals the set $H_{A, x}(Y)=\left\{f(x) \mid f \in \operatorname{Hom}_{R}(A, Y)\right\}$. Of course, every matrix subgroup is a left submodule of $Y$ over the endomorphism ring $\operatorname{End}_{R} Y$. Moreover, the functor $Y \mapsto \mathrm{H}_{A, x}(Y)$ commutes with products and coproducts.

We can measure the gap between endocoperfectness and $\Sigma$-pure-injectivity by comparing Corollary 2.6 with the following result.

Proposition 3.1. The following statements are equivalent.

(1) $M$ is $\Sigma$-pure-injective.

(2) $M^{I}$ is coperfect over End $M^{I}$ for every index set $I$.

(3) $M^{I}$ has a perfect decomposition for every index set $I$.

Proof. (1) $\Rightarrow(3)$ : Since $M^{I}$ is then $\Sigma$-pure-injective for every index set $I$, the claim follows from Example 1.5(1). Moreover, (3) $\Rightarrow(2)$ is an application of Theorem 1.1.

$(2) \Rightarrow(1)$ : We use an argument due to W. Zimmermann [40]. Let

$$
M \supseteq U_{1} \supseteq U_{2} \supseteq \ldots
$$

be a descending chain of matrix subgroups of $M$. It is well known that every matrix subgroup $U=\mathrm{H}_{A, x}(M)$ of $M$ can be written in the form $\mathrm{H}_{M^{M}, y}(M)$ by taking the element $y=\left(y_{m}\right)_{m \in M} \in M^{M}$ defined by $y_{m}=m$ if $m \in$ $\mathrm{H}_{A, x}(M)$ and $y_{m}=0$ otherwise, see for instance [36, p. 241]. But then $U^{M}=$ 
$\left(\mathrm{H}_{M^{M}, y}(M)\right)^{M}=\mathrm{H}_{M^{M}, y}\left(M^{M}\right)=$ End $M^{M} y$ is a cyclic End $M^{M}$-submodule of $M^{M}$. So, the descending chain

$$
M^{M} \supseteq U_{1}^{M} \supseteq U_{2}^{M} \supseteq \ldots
$$

is stationary, and this shows that the original chain is also stationary.

Next, we remind that by Theorem 1.4 a module $M$ has a perfect decomposition if and only if for every totally ordered direct limit in Add $M$ the canonical epimorphism $\pi: \bigoplus_{i \in I} M_{i} \longrightarrow \lim M_{i}$ is a split epimorphism. We don't know whether this is true when $M$ is $\Sigma$-coperfect over its endomorphism ring. But at least we can show that for such $M$ the pure epimorphism $\pi$ is even a $\mathscr{C}$-pure epimorphism where $\mathscr{C}$ is the class of finitely generated $R$-modules.

We first need some preliminary results. Recall that if $\mathscr{C} \subseteq \operatorname{Mod} R$ is a class of modules, then an epimorphism $p: M \rightarrow N$ in $\operatorname{Mod} R$ is called $\mathscr{C}$-pure provided $\operatorname{Hom}_{R}(C, p): \operatorname{Hom}_{R}(C, M) \longrightarrow \operatorname{Hom}_{R}(C, N)$ is an epimorphism for every $C \in \mathscr{C}$. We start with an elementary observation, whose proof we leave to the reader:

Lemma 3.2. Let $p: X \rightarrow Y$ be an epimorphism in $\operatorname{Mod} R$ and $\mathscr{C}$ be a class of modules closed under quotients. If $p$ is a $\mathscr{C}$-pure epimorphism, then the inclusion $\operatorname{Ker}(p) \hookrightarrow \operatorname{Ker}(p)+Z$ is a split monomorphism for every submodule $Z$ of $X$ belonging to $\mathscr{C}$.

If $\mathscr{C}$ is the class of $r$-generated modules for some integer $r$, then also the converse implication holds true.

The arguments in the proof of the following lemma were given to us by P. Guil Asensio.

Lemma 3.3. Let $M$ be an $R$-module, and $X$ a finitely generated submodule of $M$. Let moreover $\left(N_{i}\right)_{i \in I}$ be a chain of direct summands of $M$ with $N=$ $\bigcup_{i \in I} N_{i}$. The following assertions are equivalent:

(1) The inclusion $N \hookrightarrow N+X$ is a split monomorphism.

(2) There is an index $j \in I$ such that $X \cap N \subseteq N_{j}$.

Moreover, $M$ and $X$ satisfy the above equivalent conditions for every chain of direct summands if and only if they do so for every countable chain of direct summands.

Proof. (1) $\Rightarrow$ (2): Let $f: N+X \rightarrow N$ be a retraction for the inclusion $N \hookrightarrow N+X$. Then $g=f_{\mid X}: X \longrightarrow N$ is an $R$-homomorphism such that $g(x)=x$ for all $x \in X \cap N$. But since $X$ is finitely generated $\operatorname{Im}(g) \subseteq N_{j}$ for some $j \in I$. Then $X \cap N \subseteq N_{j}$ as desired. 
(2) $\Rightarrow$ (1): Suppose $X \cap N \subseteq N_{j}$ and let us fix a retraction $\pi: M \longrightarrow N_{j}$ for the inclusion $N_{j} \hookrightarrow M$. Then the assignment $n+x \mapsto n+\pi(x)$ gives a well-defined morphism $N+X \longrightarrow N$ which is a retraction for the canonical inclusion $N \hookrightarrow N+X$.

For the final statement we only need to prove that if condition 2) holds for every countable chain of direct summands, then it also holds for an arbitrary one. Suppose then that condition 2) holds for countable chains and let $\left(N_{i}\right)_{i \in I}$ be an arbitrary chain of direct summands of $M$. Suppose that $X \cap\left(\bigcup_{i \in I} N_{i}\right)$ is not contained in any $N_{j}$. Then the set $\left\{X \cap N_{i}: i \in I\right\}$ does not have a maximal element, and we can find a strictly ascending chain $X \cap N_{i_{1}} \subset X \cap N_{i_{2}} \subset \ldots$. So, we get a countable chain $\left(N_{i_{k}}\right)_{k=1,2, \ldots}$ of direct summands of $M$ such that $X \cap\left(\bigcup_{k>0} N_{i_{k}}\right)$ is not contained in $X \cap N_{i_{l}}$ for any $l=1,2, \ldots$, which is a contradiction.

Let $r>0$ be an integer, and $\mathscr{C}$ be the class of $r$-generated $R$-modules. We now want to describe when the canonical epimorphism $\pi: \bigoplus_{i \in I} M_{i} \longrightarrow$ $\underset{\lim }{\rightarrow} M_{i}$ associated to a direct system $\left(M_{i}, f_{j i}\right)$ is $\mathscr{C}$-pure. To this end, we


$\left(x_{1}, \ldots, x_{r}\right) \in M^{r}$, we write $a(\underline{x})=\left\{\left(a_{1}, \ldots, a_{r}\right) \in R^{r} \mid \sum_{1 \leq i \leq r} x_{i} \bar{a}_{i}=\right.$ $0\}$. Obviously, this is an $R$-submodule of $R^{r}$ with $a(\underline{x})=\operatorname{ann}_{R}(x)$ when $r=1$. Also, if $f: M \longrightarrow N$ is an $R$-homomorphism, we denote $f(\underline{x})=$ $\left(f\left(x_{1}\right), \ldots, f\left(x_{r}\right)\right) \in N^{r}$.

Proposition 3.4. Let $\left(M_{i}, f_{j i}\right)$ be a direct system of $R$-modules, and denote by $\varphi_{i}: M_{i} \rightarrow \lim M_{i}$ the canonical map. Let further $r>0$ be an integer, and $\mathscr{C}$ be the class of $r$-generated $R$-modules. The following statements are equivalent.

(1) The canonical epimorphism $\pi: \bigoplus_{i \in I} M_{i} \longrightarrow \lim _{\longrightarrow} M_{i}$ is $\mathscr{C}$-pure.

(2) For every index $j \in I$ and every $r$-generated submodule $X \subseteq M_{j}$, there is an index $n>j$ such that the composition $f_{n j}(X) \hookrightarrow M_{n} \stackrel{\varphi_{n}}{\longrightarrow} \lim _{\longrightarrow} M_{i}$ is a monomorphism.

(3) For every index $j \in I$ and every $\underline{x} \in M_{j}^{r}$, the set $\left\{a\left(f_{k j}(\underline{x})\right) \mid k \in\right.$ $I, j \leq k\}$ of submodules of $R_{R}^{r}$ has a maximal element.

Proof. Denote by $\epsilon_{i}: M_{i} \rightarrow \bigoplus_{i \in I} M_{i}$ the canonical inclusion, so $\varphi_{i}=$ $\pi \epsilon_{i}$.

(1) $\Rightarrow(2)$ : Let $X$ be an $r$-generated submodule of $M_{j}$. Then $Y=\varphi_{j}(X)$ is a $r$-generated submodule of $\lim _{\rightarrow} M_{i}$. By hypothesis, there is a morphism $h: Y \longrightarrow \bigoplus_{i \in I} M_{i}$ such that $\overrightarrow{\pi h}$ is the canonical inclusion $Y \hookrightarrow \underset{\lim }{\longrightarrow} M_{i}$. There is a finite subset $K \subseteq I$ such that $h(Y) \subseteq \bigoplus_{k \in K} M_{k}$. It is not restrictive to assume that one of the indices from $K$, say $l$, is the largest one, i.e., $k \leq l$ 
for all $k \in K$. Let $g: \bigoplus_{k \in K} M_{k} \longrightarrow M_{l}$ be the homomorphism with the components $\left(f_{l k}\right)_{k \in K}$.

We claim that the composition $Y \stackrel{h}{\longrightarrow} \bigoplus_{k \in K} M_{k} \stackrel{g}{\longrightarrow} M_{l} \stackrel{\varphi_{l}}{\longrightarrow} \underset{\lim }{\longrightarrow} M_{i}$ is the inclusion map. Indeed, if $y \in Y$ and $h(y)=\left(z_{k}\right)_{k \in K}$ with $\vec{z}_{k} \in$ $M_{k}$, then $\varphi_{l} g h(y)=\varphi_{l}\left(\sum_{k \in K} f_{l k}\left(z_{k}\right)\right)=\sum_{k \in K} \pi \epsilon_{l} f_{l k}\left(z_{k}\right)$. By Lemma 1.2 $\sum_{k \in K}\left(\epsilon_{k}\left(z_{k}\right)-\epsilon_{l} f_{l k}\left(z_{k}\right)\right) \in \operatorname{Ker} \pi$, hence $\varphi_{l} g h(y)=\sum_{k \in K} \pi \epsilon_{k}\left(z_{k}\right)=$ $\pi h(y)=y$, as desired.

Thus for any $x \in X$ we have $\varphi_{l}\left(f_{l j}(x)\right)=\varphi_{j}(x)=\varphi_{l} g h\left(\varphi_{j}(x)\right)$, hence $f_{l j}(x)-g h\left(\varphi_{j}(x)\right)$ belongs to $\operatorname{Ker}\left(\varphi_{l}\right)$, and by a well-known property of direct limits, there is an index $m>l$ in $I$ such that $f_{m l}\left(f_{l j}(x)-g h\left(\varphi_{j}(x)\right)\right)=0$. Since $X$ is finitely generated, taking $m$ large enough we obtain the latter equality for all $x \in X$, so $f_{m j}(X)=f_{m l} g h(Y)$. Now the fact that $\varphi_{l} g h(y)=y$ for all $y \in Y$ implies that the restriction of $\varphi_{m}$ on $f_{m j}(X)$ induces a split epimorphism $\tilde{\varphi}_{m}$ : $f_{m j}(X) \rightarrow Y$. Then we can decompose $f_{m j}(X)=U \oplus V$ in such a way that the restriction of $\left.\tilde{\varphi}_{m}\right|_{U}: U \stackrel{\cong}{\longrightarrow} Y$ is an isomorphism, while $\varphi_{m}(V)=0$. Since $V$ is finitely generated, we see as above that there is an index $n>m$ in $I$ such that $f_{n m}(V)=0$. So, if we factor the monomorphism $\left.\varphi_{m}\right|_{U}$ through $f_{n m}$, we obtain $\left.\varphi_{m}\right|_{U}: U \stackrel{\left.f_{n m}\right|_{U}}{\longrightarrow} f_{n m}(U)=f_{n m} f_{m j}(X)=f_{n j}(X) \hookrightarrow M_{n} \stackrel{\varphi_{n}}{\longrightarrow} \underline{\lim _{\longrightarrow}} M_{i}$ where the first map is an isomorphism. Hence the restriction $\left.\varphi_{n}\right|_{f_{n j}(X)} \overrightarrow{\text { is a }}$ monomorphism.

(2) $\Rightarrow(1)$ : We need to prove that if $Y \subset \underset{\lim }{\longrightarrow} M_{i}=: M^{\prime}$ is an $r$-generated submodule, then the canonical inclusion $Y \overleftrightarrow{\longrightarrow} \lim M_{i}$ factors through $\pi$ : $\bigoplus_{i \in I} M_{i} \longrightarrow \lim _{\longrightarrow} M_{i}$. Since $M^{\prime}=\bigcup_{i \in I} \varphi_{i}\left(M_{i}\right)$ (directed union), there is a $j \in I$ such that $\vec{Y} \subset \varphi_{j}\left(M_{j}\right)$, which implies the existence of an $r$-generated submodule $X \subset M_{j}$ such that $\varphi_{j}(X)=Y$. By hypothesis, there is an index $n>$ $j$ such that the composition $f_{n j}(X) \hookrightarrow M_{n} \stackrel{\varphi_{n}}{\longrightarrow} \lim _{\longrightarrow} M_{i}$ is a monomorphism. This means that $\varphi_{n}$ induces by restriction an isomorphism $q: f_{n j}(X) \stackrel{\cong}{\longrightarrow} Y$. We now consider the composition $h: Y \stackrel{q^{-1}}{\longrightarrow} f_{n j}(X) \hookrightarrow M_{n} \stackrel{\epsilon_{n}}{\longrightarrow} \bigoplus_{i \in I} M_{i}$, and easily check that $\pi h$ is just the canonical inclusion $Y \hookrightarrow \lim _{\longrightarrow} M_{i}$.

(2) $\Leftrightarrow$ (3): Condition (2) holds if and only if for every index $\overrightarrow{j \in I}$ and every $r$-generated submodule $X \subseteq M_{j}$, there is an index $k>j$ such that the structural map $f_{l k}: M_{k} \longrightarrow M_{l}$ induces an isomorphism $\widetilde{f_{l k}}: f_{k j}(X) \stackrel{\cong}{\cong} f_{l j}(X)$ for all $l \geq k$. If we take a set $\left\{x_{1}, \ldots, x_{r}\right\}$ of $r$ generators of $X$ and put $\underline{x}=\left(x_{1}, \ldots, x_{r}\right) \in M_{j}{ }^{r}$, then $a\left(f_{k j}(\underline{x})\right) \subseteq a\left(f_{l j}(\underline{x})\right)$ whenever $j \leq k \leq l$, and equality holds if and only if Ker $\widetilde{f_{l k}}=0$. Now the equivalence of conditions (2) and (3) follows easily.

We now draw some consequences. 
Corollary 3.5. Let $M$ be an an R-module, $r>0$ an integer, and let $\left(N_{i}\right)_{i \in I}$ be a chain of direct summands of $M$ with $N=\bigcup_{i \in I} N_{i}$. Suppose that for every sequence $M \stackrel{f_{1}}{\longrightarrow} M \stackrel{f_{2}}{\longrightarrow} M \stackrel{f_{3}}{\longrightarrow} \ldots$ of $R$-homomorphisms and every $\underline{x} \in M^{r}$, the set $\left\{a\left(f_{n} \cdots f_{1}(\underline{x})\right) \mid n \in \mathrm{N}\right\}$ has a maximal element. Then the inclusion $N \hookrightarrow N+X$ is a split monomorphism for every r-generated submodule $X \subseteq M$.

Proof. By Lemma 3.3, we can assume that the chain is countable and, hence, that $I=\mathrm{N}$. As in the proof of Proposition 1.3, we form a sequence $M \stackrel{f_{1}}{\longrightarrow} M \stackrel{f_{2}}{\longrightarrow} M \stackrel{f_{3}}{\longrightarrow} \ldots$ of idempotent morphisms such that $\operatorname{Im}\left(1-f_{i}\right)=$ $N_{i}$ and $f_{j} f_{i}=f_{j}$ whenever $i \leq j$, and we define a direct system by taking $M_{i}=M$ and $f_{j i}=f_{j}$ for $i<j$. Let $\pi: M^{(\mathrm{N})} \rightarrow \underset{\lim }{\longrightarrow} M_{i}$ be the canonical epimorphism, and $\nabla: M^{(\mathrm{N})} \longrightarrow M,\left(x_{i}\right) \mapsto \sum x_{i}$ the summation map. Note that $\operatorname{Ker} \pi=\operatorname{Im}(1-f)$ where $f$ is defined as in the proof of Proposition 2.1, so it is easy to check that $\operatorname{Ker}(\pi)=\left\{\left(x_{i}\right) \in M^{(\mathrm{N})} \mid \nabla\left(x_{i}\right) \in N\right\}$. Moreover, by our hypothesis and Proposition 3.4, the canonical epimorphism $\pi: M^{(\mathrm{N})} \rightarrow$ $\underset{\lim }{\longrightarrow} M_{i}$ is $\mathscr{C}$-pure, where $\mathscr{C}$ is the class of $r$-generated modules. By Lemma 3.2,

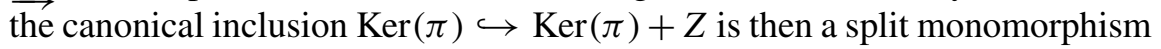
for every $r$-generated submodule $Z$ of $M^{(\mathrm{N})}$.

Suppose now that $X$ is an $r$-generated submodule of $M$. Then for a fixed $j \in \mathrm{N}$, we have $X \subseteq M_{j}$ and $\epsilon_{j}(X)$ is an $r$-generated submodule of $M^{(\mathrm{N})}$, where $\epsilon_{j}: M=M_{j} \longrightarrow M^{(\mathrm{N})}$ is the canonical inclusion. We have that $\epsilon_{j}(X) \cap \operatorname{Ker}(\pi)=\epsilon_{j}(X \cap N)$. Since $\operatorname{Ker}(\pi) \hookrightarrow \operatorname{Ker}(\pi)+\epsilon_{j}(X)$ is a split monomorphism, we get a map $g: X \longrightarrow \operatorname{Ker}(\pi)$ such that $g(x)=\epsilon_{j}(x)$ whenever $\epsilon_{j}(x) \in \operatorname{Ker}(\pi)$. That is, $g(x)=\epsilon_{j}(x)$ whenever $x \in X \cap N$. Now the composition $h: X \stackrel{g}{\longrightarrow} \operatorname{Ker}(\pi) \stackrel{\nabla}{\longrightarrow} N$ is a morphism such that $h(x)=x$ whenever $x \in X \cap N$. Then the assignment $n+x \mapsto n+h(x)$ defines an $R$-homomorphism, which is a retraction for the inclusion $N \hookrightarrow N+X$.

Proposition 3.6. Let $M$ be an $R$-module, $r>0$ an integer, and $\mathscr{C}$ the class of $r$-generated $R$-modules. If $M$ is $r$-coperfect over $S=\operatorname{End}_{R}(M)$, then the following assertions hold true:

(1) For every totally ordered direct system $\left(M_{i}, f_{j i}\right)$ in Add $M$, the canonical epimorphism $\pi: \bigoplus_{i \in I} M_{i} \longrightarrow \lim _{\longrightarrow} M_{i}$ is $\mathscr{C}$-pure.

(2) If $M^{\prime} \in \operatorname{Add} M$, and $\left(N_{i}\right)_{i \in I}$ is a chain of direct summands of $M^{\prime}$ with $N=\bigcup_{i \in I} N_{i}$, then the inclusion $N \hookrightarrow N+X$ is a split monomorphism for every $r$-generated submodule $X$ of $M^{\prime}$.

Proof. We first prove assertion (1) in case $I=\mathrm{N}$ is countable. There is no loss of generality in assuming that there is a set $A$ such that $M_{i}=M^{(A)}$ for all $i \in \mathrm{N}$. Then the direct system comes from a sequence of endomorphisms 
$M^{(A)} \stackrel{f_{1}}{\longrightarrow} M^{(A)} \stackrel{f_{2}}{\longrightarrow} \ldots$, and $M^{(A)}$ is $r$-coperfect over its endomorphism ring by Corollary 2.6. So, the module $M^{(A)}$ satisfies condition (3) of Proposition 2.2, which in turn implies condition (2) of Proposition 3.4. Thus $\pi: \bigoplus_{i \in \mathrm{N}} M_{i} \rightarrow$ $\lim _{i} M_{i}$ is $\mathscr{C}$-pure.

Combining Proposition 3.4 with Corollary 3.5, we now obtain assertion (2). Finally, in order to prove assertion (1) in the general case, we recall from Lemma 1.2 that $\operatorname{Ker}(\pi)$ is the union of a chain of direct summands of $\bigoplus_{i \in I} M_{i}$. The result then follows from assertion (2) and the second part of Lemma 3.2.

We now obtain the announced result as an immediate consequence.

COROLlaRy 3.7. If $M$ is $\Sigma$-coperfect over its endomorphism ring, then for every totally ordered direct system $\left(M_{i}, f_{j i}\right)$ in Add $M$ the canonical epimorphism $\pi: \bigoplus_{i \in I} M_{i} \rightarrow \lim _{\longrightarrow} M_{i}$ is $\mathscr{C}$-pure, where $\mathscr{C}$ is the class of finitely generated $R$-modules.

\section{Finitely generated endocoperfect modules}

We now prove that Question 1 has a positive answer for finitely generated modules.

We first need two preliminary results. We have discussed in Section 2 how endocoperfectness behaves with respect to direct sums. As for direct summands, it is straightforward to verify the following result.

Lemma 4.1. If $r \in \mathrm{N}$ and $M$ is a module which is $r$-coperfect over End $M$, then every direct summand $N$ of $M$ is $r$-coperfect over End $N$.

Lemma 4.2. Let $M$ be a module which is coperfect over $S=$ End $M$. Assume that $M$ is finitely generated, or more generally, that there is a finite subset $X=\left\{x_{1}, \ldots, x_{r}\right\} \subseteq M$ such that $\operatorname{ann}_{S}(M)=\operatorname{ann}_{S}(X)$. Then $S$ is a left semiartinian ring, and the Jacobson radical $J(S)$ is left T-nilpotent. Moreover, if $M$ is indecomposable or $\Sigma$-coperfect over $S$, then $S$ is a right perfect ring.

Proof. By the assumption on $M$ we have an embedding $\lambda:{ }_{S} S \longrightarrow$ ${ }_{s} M^{r}, f \mapsto\left(f\left(x_{1}\right), \ldots, f\left(x_{r}\right)\right)$. Since ${ }_{S} M$ is coperfect, ${ }_{s} M^{r}$ is semiartinian. Then $S$ is a left semiartinian ring, and $J(S)$ is left T-nilpotent, see [32, Prop. VIII.2.6]. Furthermore, if $M$ is indecomposable, then $S$ has the only idempotents 0 and 1 and is thus right perfect by [25, 11.6.3]. Finally, if $M \Sigma$ coperfect over $S$, then the above embedding $\lambda$ shows that $S$ satisfies dec on cyclic left ideals, hence $S$ is right perfect also in this case.

Theorem 4.3. Let $M$ be a module with $S=$ End $M$. Assume that $M$ is finitely generated, or more generally, that there is a finite subset $X \subseteq M$ such that $\operatorname{ann}_{S}(M)=\operatorname{ann}_{S}(X)$. Then the following statements are equivalent. 
(1) ${ }_{S} M$ is $\Sigma$-coperfect.

(2) $S$ is right perfect.

(3) $M$ has an indecomposable decomposition and ${ }_{S} M$ is coperfect.

(4) $M$ has a perfect decomposition.

(5) If $M \stackrel{f_{1}}{\longrightarrow} M \stackrel{f_{2}}{\longrightarrow} M \stackrel{f_{3}}{\longrightarrow} \ldots$ is a sequence of endomorphisms, and $\left(M_{n}, f_{n m}\right)_{\mathrm{N}}$ is the direct system given by $M_{n}=M, f_{n m}=1_{M_{n}}$ if $n=m$, and $f_{n m}=f_{n-1} \ldots f_{m}$ if $n>m$, then the canonical epimorphism $\pi$ : $\bigoplus_{n \in \mathrm{N}} M_{n} \longrightarrow \lim _{\longrightarrow} M_{n}$ is a split epimorphism.

Proof. (1) $\Rightarrow(2),(3): S$ is a right perfect ring by Lemma 4.2, and $M$ has then an indecomposable decomposition, see [17, 3.14]. For $(2) \Rightarrow(1)$ we refer to [25, Cor. 11.7.2].

(3) $\Rightarrow(4)$ : By Lemma 4.1 and Theorem 1.1 we can assume that $M_{R}$ is indecomposable and only have to verify that $S=$ End $M$ is local. This follows immediately from Lemma 4.2.

Finally, Theorem 1.4 yields $(4) \Rightarrow(5)$, and Propositions 2.1 and 2.2 give (5) $\Rightarrow(1)$.

We now apply Theorem 4.3 to exhibit some cases in which endocoperfectness already entails a perfect decomposition.

EXAMPLES 4.4. (1) A finitely generated pure-injective module $M$ has a perfect decomposition if and only if it is endocoperfect.

Indeed, if $S=$ End $M$, then the pure-injectivity of $M$ implies that $S / J(S)$ is right self-injective and von Neumann regular [37, Theorem 9]. Moreover, if ${ }_{S} M$ is coperfect, then $S / J(S)$ is semiartinian and $J(S)$ is left T-nilpotent by Lemma 4.2. From [6, Cor. 4.6] it follows that $S / J(S)$ is semisimple. Thus $S$ is right perfect.

(2) Let $M_{R}$ be a module which is a finite sum of cyclic invariant submodules. Then $M$ has a perfect decomposition if and only if it is endocoperfect.

In fact, in this case $M=x_{1} R+\cdots+x_{r} R$ where each $x_{i} R$ is also an $S$ submodule of $M$ for $S=$ End $M$. In particular, $\operatorname{ann}_{S}\left(x_{i}\right)=\left\{f \in S \mid f\left(x_{i}\right)=\right.$ $0\}$ is then a two-sided ideal for each $1 \leq i \leq r$. So, if ${ }_{S} M$ is coperfect, we infer from ${ }_{S} S / \operatorname{ann}_{S}\left(x_{i}\right) \cong{ }_{S} S x_{i}$ that $S / \operatorname{ann}_{S}\left(x_{i}\right)$ is a right perfect ring for any $i=$ $1, \ldots, r$, and we conclude by $\left[9\right.$, Lemma 2.6] that $S /\left(\operatorname{ann}_{S}\left(x_{1}\right) \cap \ldots \cap \operatorname{ann}_{S}\left(x_{r}\right)\right)$ is right perfect. But $\operatorname{ann}_{S}\left(x_{1}\right) \cap \ldots \cap \operatorname{ann}_{S}\left(x_{r}\right)=0$, hence $S$ is right perfect.

(3) Let $M_{R}$ be a finite direct sum of cyclic modules. Then $M$ has a perfect decomposition if and only if it is endocoperfect.

Indeed, if $M$ is endocoperfect then each of the cyclic summands of $M$ is endocoperfect by Lemma 4.1, and then, by the foregoing example, has a 
perfect decomposition. In particular, $M$ is then a direct sum of modules with local endomorphism ring. Now apply Theorem 1.1.

(4) Let $R$ be a Noether algebra, that is, an algebra which is finitely generated as a module over its noetherian center $K$. Then a finitely generated $R$-module has a perfect decomposition if and only if it is endocoperfect.

Indeed, if $M_{R}$ is finitely generated, then $S=\operatorname{End}_{R}(M)$ is also a Noether $K$-algebra, thus $S$ is left and right noetherian. Moreover, if ${ }_{S} M$ is coperfect, then $S$ is also left semiartinian by Lemma 4.2, so we conclude that $S$ is left artinian and hence right perfect.

Next, we briefly discuss the relationship between perfect decompositions and the existence of Add $M$-covers. Here we adopt the terminology of [16]. Notice that covers are also called minimal right approximations.

REMARK 4.5. It is well known that a class of the form Add $M$ is always precovering. If $M$ has a perfect decomposition, then Add $M$ is even a covering class [2, 4.1]. The converse implication holds true in case that $M$ is a direct sum of finitely presented modules $[2,4.4]$. The following is a further case where the converse implication holds true.

ExAmple 4.6. Assume that $M$ is a $*$-module in the sense of [11], or more generally, that $M$ is a finitely generated module such that the functor $\operatorname{Hom}_{R}(M,-)$ is exact on any pure-exact sequence consisting of $M$-generated modules. Then $M$ has a perfect decomposition if and only if the class Add $M$ is covering.

In fact, since $M$ is finitely generated, the covariant functor $\operatorname{Hom}_{R}(M,-)$ : $\operatorname{Mod} R \longrightarrow \operatorname{Mod} S$ induces an equivalence between Add $M$ and the category of projective $S$-modules and turns Add $M$-covers into projective covers. So, every right $S$-module of the form $\operatorname{Hom}(M, X)$ for some $X \in \operatorname{Mod} R$ has a projective cover.

Let us now verify condition (5) in Theorem 4.3. Let $M \stackrel{f_{1}}{\longrightarrow} M \stackrel{f_{2}}{\longrightarrow} M$ $\stackrel{f_{3}}{\longrightarrow} \ldots$ be a sequence of endomorphisms. We apply the functor $H=$ $\operatorname{Hom}_{R}(M,-)$ and consider the endomorphisms $f_{i}^{*}=H\left(f_{i}\right): S \rightarrow S$ acting on $S$ as left multiplication by $f_{i}$. We obtain the following commutative diagram with exact rows

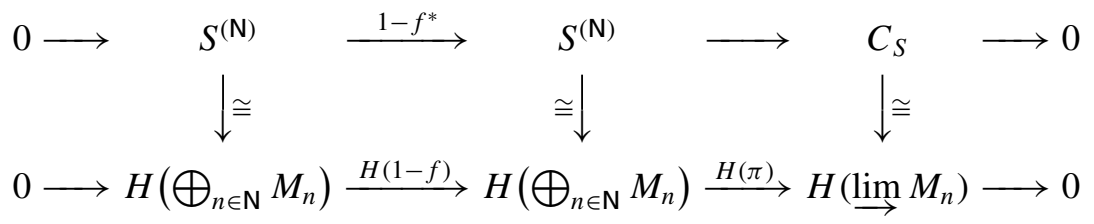


where the maps $1-f$ and $\pi$ in the second row are defined as in the proof of Proposition 2.1, and the map $1-f^{*}$ in the first row is defined as $1_{S^{(\mathrm{N})}}-f^{*}$ with $f^{*}$ the $S$-homomorphism given by the matrix

$$
\left(\begin{array}{cccc}
0 & \ldots & & \\
f_{1}^{*} & 0 & \ldots & \\
0 & f_{2}^{*} & 0 & \ldots \\
\vdots & 0 & f_{3}^{*} & \ddots \\
& \vdots & \ddots & \ddots
\end{array}\right)
$$

By a well-known argument of Bass [7] we know that $C_{S}$ is flat. We then deduce that $C_{S}$ is projective since it has a projective cover by the above considerations. So the above sequences split, and applying $-\otimes_{S} M$, we obtain a commutative diagram

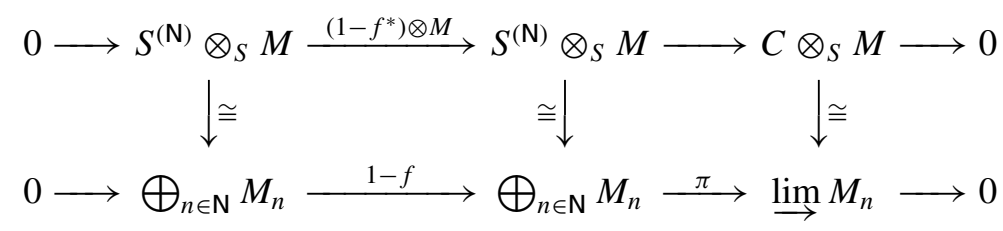

from which we infer that $\pi$ splits.

Let us now push the arguments in Theorem 4.3 a little further.

Theorem 4.7. Let $M$ be a module with $S=$ End $M$. Assume that $M$ is a direct sum of finitely generated modules. Then the following statements are equivalent.

(1) ${ }_{S} M$ is $\Sigma$-coperfect.

(2) $M$ has a perfect decomposition.

(3) $\operatorname{End}_{R}\left(M^{(\aleph)}\right)$ is von Neumann regular modulo its Jacobson radical for every cardinal $\aleph$.

(4) $\operatorname{End}_{R}\left(M^{\left(\aleph_{0}\right)}\right)$ is von Neumann regular modulo its Jacobson radical.

Proof. Write $M=\bigoplus_{i \in I} M_{i}$ with finitely generated modules $M_{i}$.

$(1) \Rightarrow(2)$ : Applying Lemma 4.1 and Theorem 4.3 to all indices $i \in I$, we get a decomposition $M=\oplus_{j \in J} X_{j}$ for some family $\left(X_{j}\right)_{j \in J}$ of indecomposable modules with local endomorphism ring. That this family is right $T$-nilpotent follows from [22, Prop. E].

(2) $\Rightarrow$ (1) holds by Corollary 2.3, and (2) $\Rightarrow$ (3) by Theorem 1.1. (3) $\Rightarrow$ (4) is obvious. So, it remains to prove (4) $\Rightarrow(2)$ : Let us denote $A=\operatorname{End}_{R}\left(M^{\left(\aleph_{0}\right)}\right)$. 
Since $\operatorname{End}_{R}\left(M_{i}^{\left(\aleph_{0}\right)}\right)$ is of the form $e A e$ for some idempotent $e \in A$, we have that $\operatorname{End}_{R}\left(M_{i}^{\left(\aleph_{0}\right)}\right)$ is also von Neumann regular modulo its Jacobson radical. But, since $M_{i}$ is $R$-finitely generated, $\operatorname{End}_{R}\left(M_{i}^{\left(\aleph_{0}\right)}\right)$ is isomorphic to the ring of column-finite $\mathrm{N} \times \mathrm{N}$-matrices with entries in $\operatorname{End}_{R}\left(M_{i}\right)$. By [12, Theorem 1] we infer that $\operatorname{End}_{R}\left(M_{i}\right)$ is a right perfect ring, for all $i \in I$. Arguing as in implication (1) $\Rightarrow(2)$, we conclude that $M_{R}$ is a direct sum of indecomposables with local right perfect endomorphism ring. There is no loss of generality in assuming from now on that all the $M_{i}$ are indecomposable with local right perfect endomorphism ring. Then, we need to prove that the family $\left(M_{i}\right)_{i \in I}$ is right T-nilpotent, for which we adapt the argument in the proof of [12, Prop. 1]. Let $M_{i_{1}} \stackrel{f_{1}}{\longrightarrow} M_{i_{2}} \stackrel{f_{2}}{\longrightarrow} M_{i_{3}} \stackrel{f_{3}}{\longrightarrow} \ldots$ be a sequence of non-isomorphisms, possibly with some of the indices $i_{k}$ repeated. We introduce the new set of indices $\Lambda=I \times \mathrm{N}$ and define $M_{(i, n)}=M_{i}$ for all $(i, n) \in \Lambda$. Now $M^{\left(\aleph_{0}\right)}=$ $\oplus_{(i, n) \in \Lambda} M_{(i, n)}$ and every element of $A$ can be identified with a column-finite $\Lambda \times \Lambda$-matrix $\left(f_{[(i, m),(j, n)]}\right)$, where $f_{[(i, m),(j, n)]} \in \operatorname{Hom}_{R}\left(M_{j}, M_{i}\right)$ for all pairs $[(i, m),(j, n)]$. We choose $f \in A$ such that its $\left[\left(i_{k+1}, k+1\right),\left(i_{k}, k\right)\right]$-entry is $f_{k}$ for every $k=1,2, \ldots$, while the remaining entries are zero. Since $A / J(A)$ is von Neumann regular, there is a $g \in A$ such that $f-f g f \in J(A)$. Then the $\left[\left(i_{k+1}, k+1\right),\left(i_{k}, k\right)\right]$-entry of $f-f g f$ is $f_{k}-f_{k} g_{k} f_{k}$, where $g_{k}$ : $M_{i_{k+1}} \longrightarrow M_{i_{k}}$ is the $\left[\left(i_{k}, k\right),\left(i_{k+1}, k+1\right)\right]$-entry of $g$. But, since $f_{k}$ is not an isomorphism, $g_{k} f_{k}$ is in the Jacobson radical of $\operatorname{End}_{R}\left(M_{i_{k}}\right)$ and, hence, there is a $h_{k} \in \operatorname{End}_{R}\left(M_{i_{k}}\right)$ such that $\left(1_{M_{i_{k}}}-g_{k} f_{k}\right) h_{k}=1_{M_{i_{k}}}$. If we now take the $h \in A$ whose $\left[\left(i_{k}, k\right),\left(i_{k}, k\right)\right]$-entry is $h_{k}$ for all $k=1,2, \ldots$, and the remaining entries are zero, then direct calculation shows that $(f-f g f) h$ is an element of $J(A)$ whose $\left[\left(i_{k+1}, k+1\right),\left(i_{k}, k\right)\right]$-entry is $f_{k}$ for all $k=1,2, \ldots$ By using Zelmanowitz's criterion from [35, Corollary 1], which is valid here since all the $M_{(i, n)}$ are finitely generated, we conclude that $f_{n} \ldots f_{1}=0$ for $n$ large enough.

As an application, we obtain a new characterization of right pure-semisimple rings, which is related to results in [34], [27], [2]. Recall that $R$ is said to be right pure-semisimple if every right $R$-module is pure-injective.

Corollary 4.8. Let $R$ be any ring, $\left\{M_{i} \mid i \in I\right\}$ be a family of representatives, up to isomorphism, of the finitely presented right $R$-modules and put $M=\oplus_{i \in I} M_{i}$. The following assertions are equivalent:

(1) $R$ is a right pure-semisimple ring.

(2) $\operatorname{End}_{R}\left(M^{\left(\aleph_{0}\right)}\right)$ is von Neumann regular modulo its Jacobson radical.

Proof. $(1) \Rightarrow(2)$ : Since $M^{\left(\aleph_{0}\right)}$ is a pure-injective module, the implication follows from the well-known fact that pure-injective modules have semiregular endomorphism ring. 
$(2) \Rightarrow(1)$ : In this case, every finite matrix subgroup is clearly a finitely generated endosubmodule of $M$, for details see [2, Section 3]. Since, by Theorem 4.7, ${ }_{S} M$ is coperfect, we conclude that $M_{R}$ is $\Sigma$-pure-injective. But then every pure-projective right $R$-module, as an object of Add $M$, is pure-injective. Therefore $R$ is right pure-semisimple (cf. [26, Theorem 2.1])

\section{Endocoperfect CS-modules}

This last section is devoted to another case where endocoperfectness already entails a perfect decomposition, namely the case in which $M_{R}$ is a CS-module. Recall that a module $M_{R}$ is said to be a CS-module (or an extending module) if every submodule $U$ of $M$ is an essential submodule of some direct summand $N$ of $M$. We further say that a submodule $N$ of $M$ is an essentially closed submodule if it has no proper essential extensions in $M$. We can then rephrase the above definition by saying that $M$ is a CS-module if and only if every essentially closed submodule is a direct summand.

The investigations in Section 3 will be very useful in this context. In fact, the following is a straightforward observation.

REMARK 5.1. Let $M$ be a module and $N$ a submodule of $M$. If the inclusion $N \hookrightarrow N+X$ is a split monomorphism for every cyclic submodule $X \subseteq M$, then $N$ is essentially closed in $M$.

So, as a first consequence of Corollary 3.5, we rediscover the following result.

COROLlaRY 5.2 (Okado). If $M$ is a CS-module over a ring $R$ satisfying the ascending chain condition on ideals of the form $\operatorname{ann}_{R}(x)$ with $x \in M$, then $M$ is a direct sum of uniform modules.

Proof. The CS-condition and Corollary 3.5 imply that the union of every chain of direct summands of $M$ is a direct summand. Then [28, Lemma 2.16 and Theorem 2.17] yield that $M$ is a direct sum of indecomposables, which are necessarily uniform.

Similarly, the following is an immediate consequence of Proposition 3.6.

Corollary 5.3. If $M_{R}$ is an endocoperfect CS-module, then $M$ is a direct sum of uniform modules.

We now want to show that endocoperfect CS-modules even have a perfect decomposition.

LeMmA 5.4. Let $M$ be a module which is coperfect over $S=$ End $M$.

(1) For each $f \in S$ and each $x \in M$ there are $n \in \mathrm{N}$ and $g \in S$ such that $(1-g f)\left(f^{n}(x)\right)=0$. 
(2) Let $M$ be uniform and $f \in S$. Then $f$ is not a monomorphism if and only if $M=\bigcup_{n \in \mathrm{N}} \operatorname{Ker} f^{n}$.

Proof. (1) follows immediately from Proposition 2.2.

(2) The if-part is clear. For the only-if-part, we assume that there is an $x \in M$ such that $f^{n}(x) \neq 0$ for all $n \in \mathrm{N}$. Then by statement (1) there is $g \in S$ such that $1-g f$ is not a monomorphism. Since $\operatorname{Ker}(1-g f) \cap \operatorname{Ker} g f=0$ and $M$ is uniform, we infer that $g f$ and $f$ are monomorphisms.

THEOREM 5.5. A CS-module has a perfect decomposition if and only if it is coperfect over its endomorphism ring.

Proof. By Theorem 1.1 we have to show that every endocoperfect CSmodule has a decomposition in modules with local endomorphism ring. In view of Corollary 5.3 and Lemma 4.1, it only remains to prove that the endomorphism ring $S$ of any endocoperfect uniform module $M$ is local.

We first show that $J(S)=\{f \in S \mid f$ is not a monomorphism $\}$. The inclusion $\subseteq$ follows immediately from statement (1) in Lemma 5.4. For the other inclusion, we consider $f \in S$ which is not a monomorphism and take an arbitrary $g \in S$. Then $g f$ is not a monomorphism, so Lemma 5.4 tells that $M=$ $\bigcup_{n \in \mathrm{N}} \operatorname{Ker}(g f)^{n}$. But then $h=\sum_{n \in \mathrm{N}}(g f)^{n}$ is a well-defined endomorphism which is inverse to $1-g f$. This shows that $f \in J(S)$.

Now we have only to verify that non-isomorphisms $f \in S$ cannot be monomorphisms. Indeed, if $f$ is a monomorphism, then so is $f^{n}$ for any $n \in \mathbf{N}$. So, if we choose $x \in M$ together with an integer $n \in \mathrm{N}$ and an endomorphism $g \in S$ such that $(1-g f)\left(f^{n}(x)\right)=0$, we see that $1-g f$ is not a monomorphism and therefore belongs to $J(S)$. Hence $g f=1-(1-g f)$ is invertible and $f$ is a split monomorphism. Thus $f$ is an isomorphism.

The above results, combined with the work of Gómez Pardo and Guil Asensio [19], [18], imply that every $\Sigma$-CS-module is endocoperfect. But they also yield a new class of CS-modules with perfect decomposition. In fact, endocoperfect CS-modules need not be $\Sigma$-CS, as shown by the following example.

EXAMPLE 5.6. The ring $R=\left(\begin{array}{ll}\mathrm{R} & \mathrm{C} \\ 0 & \mathrm{C}\end{array}\right)$ is two-sided artinian and right CS, but $(R \oplus R)_{R}$ is not CS, see [23]. So $R_{R}$ is an endocoperfect CS-module which is not $\Sigma-C S$.

N. V. Dung has shown in $[15,4.3]$ that if a CS-module has an indecomposable decomposition $M=\bigoplus_{k \in K} X_{k}$ that complements maximal direct summands, then the family $\left(X_{k}\right)_{k \in K}$ is locally semi-T-nilpotent. However, in general, $\left(X_{k}\right)_{k \in K}$ will not be locally T-nilpotent. In fact, there are CS-modules 
with a decomposition in modules with local endomorphism ring (hence satisfying the above assumption) that are not endocoperfect and thus do not have a perfect decomposition.

Example 5.7. The power series ring $R=K[[x]]$ over a field $K$ is a local non-artinian PID, and therefore a CS-ring by $[14,12.10]$. So $R_{R}$ is a nonendocoperfect CS-module with local endomorphism ring. This also proves that, in Okado's result (cf. Corollary 5.2), the decomposition is not perfect in general.

ACKNOWLEDGEMENTs. We thank P. Guil Asensio for helpful and stimulating conversations.

\section{REFERENCES}

1. Anderson, F. W., and Fuller, K. R., Rings and Categories of Modules, second ed., Springer, New York, 1992.

2. Angeleri Hügel, L., Covers and envelopes via endoproperties of modules, Proc. London Math. Soc. 86 (2003), 649-665.

3. Angeleri Hügel, L., and Coelho, F. U., Infinitely generated tilting modules of finite projective dimension, Forum Math. 13 (2001), 239-250.

4. Angeleri Hügel, L., and Trlifaj, J., Direct limits of modules of finite projective dimension, in Rings, Modules, Algebras, and Abelian Groups, Lecture Notes in Pure and Appl. Math. 236 (2004), 27-44.

5. Azumaya, G., Finite splitness and finite projectivity, J. Algebra 106 (1987), 114-134.

6. Baccella, G., Semiartinian $V$-rings and semiartinian von Neumann regular rings, J. Algebra 173 (1995), 587-612.

7. Bass, H., Finitistic dimension and a homological generalization of semiprimary rings, Trans. Amer. Math. Soc. 95 (1960), 466-488.

8. Björk, J. E., Rings satisfying a minimum condition on principal ideals, J. Reine Angew. Math. 236 (1969), 112-119.

9. Björk, J. E., Radical properties of perfect modules, J. Reine Angew. Math. 253 (1972), 78-86.

10. Colby, R. R., and Fuller, K. R., Tilting, cotilting, and serially tilted rings, Comm. Algebra 18 (1990), 1585-1615.

11. Colpi, R., Some remarks on equivalences between categories of modules, Comm. Algebra 18 (1990), 1935-1951.

12. Costa-Cano, F. J., and Simón, J. J., On semiregular infinite matrix rings, Comm. Algebra 27(12) (1999), 5737-5740.

13. Crawley-Boevey, W., Locally finitely presented additive categories, Comm. Algebra 22 (1994), 1641-1674.

14. Viet Dung, N., Van Huynh, D., Smith, P. F., and Wisbauer, R., Extending modules, with the collaboration of John Clark and N. Vanaja, Pitman Res. Notes Math. Ser. 313 (1994).

15. Viet Dung, N., Indecomposable decompositions that complement maximal direct summands, J. Algebra 197 (1997), 449-467.

16. Enochs, E., and Jenda, O., Relative Homological Algebra, de Gruyter, Berlin, 2000.

17. Facchini, A., Module Theory. Endomorphism rings and direct sum decompositions in some classes of modules, Prog. Math. 167 (1998). 
18. Gómez Pardo, J. L., and Guil Asensio, P., Big direct sums of copies of a module have well behaved indecomposable decompositions, J. Algebra 232 (2000), 86-93.

19. Gómez Pardo, J. L., and Guil Asensio, P., Every $\Sigma$-CS-module has an indecomposable decomposition, Proc. Amer. Math. Soc. 129 (2001), 947-954.

20. Guil Asensio, P., and Herzog, I., $\Sigma$-cotorsion rings, Adv. Math. 191 (2005), 11-28.

21. Harada, M., Factor Categories with Applications to Direct Decompositions of Modules, Lect. Notes in Pure and Appl. Math. 88 (1983).

22. Huisgen-Zimmermann, B., and Saorín, M., Direct sums of representations as modules over their endomorphism rings, J. Algebra 250 (2002), 67-89.

23. Van Huynh, D., Jain, S. K., and Lopez-Permouth, S. R., Rings characterized by direct sums of CS modules, Comm. Algebra 28 (2000), 4219-4222.

24. Jensen, C. U., Homological dimension of rings with countably generated ideals, Math. Scand. 18 (1966), 97-105.

25. Kasch, F., Modules and Rings, Academic Press (1982)

26. Kielpiński, R., and Simson, D., On pure homological dimension, Bull. Polish Acad. Sci. Math. 23 (1975), 1-6.

27. Leszczyǹski, Z., and Simson, D., On pure-semisimple Grothendieck categories and the exchange property, Bull. Polish Acad. Sci. Math. XXVII (1979), 41-46.

28. Mohamed, S. H., Müller, B., Continuous and Discrete Modules, London Math. Soc. Lecture Note Ser. 147 (1990)

29. Okado, M., On the decomposition of extending modules, Math. Japon. 29 (1984), 939-941.

30. Raynaud, M., and Gruson, L., Critères de platitude et de projectivité, Invent. Math. 13 (1971), 1-89.

31. Simson, D., On pure global dimension of locally finitely presented Grothendieck categories, Fund. Math. 96 (1977), 91-116.

32. Stenström, B., Rings of Quotients, Springer-Verlag (1975).

33. Whitehead, J. M., Projective modules and their trace ideals, Comm. Algebra 8 (1980), no. 19, 1873-1901.

34. Yamagata, K., On rings of finite representation type and modules with the finite exchange property, Sci. Rep. Tokyo Kyoiku Daigaku Sect. A 13, no. 347-365 (1975), 1-6.

35. Zelmanowitz, J., Radical endomorphisms of decomposable modules, J. Algebra 279 (2004), no. 1, 135-146.

36. Zimmermann-Huisgen, B., Pure submodules of direct products of free modules, Math. Ann. 224 (1976), 233-245.

37. Zimmermann-Huisgen, B., and Zimmermann, W., Algebraic compact rings and modules, Math. Z. 161 (1978), 81-93.

38. Zimmermann, W., Rein injektive direkte Summanden von Moduln, Comm. Algebra 5 (1977), 1083-1117.

39. Zimmermann, W., $\pi$-projektive Moduln, J. Reine Angew. Math. 292 (1977), 117-124.

40. Zimmermann, W., Lectures, University Munich 1998.

DICOM

UNIVERSITÀ DEGLI STUDI DELL'INSUBRIA

VIA MAZZINI 5, I-21100 VARESE

ITALY

E-mail: lidia.angeleri@uninsubria.it
DEPARTAMENTO DE MATEMÁTICAS

UNIVERSIDAD DE MURCIA, APTDO. 4021

E-30100 ESPINARDO, MURCIA

SPAIN

E-mail: msaorinc@um.es 